\title{
Kernos
}

Revue internationale et pluridisciplinaire de religion grecque antique

9| 1996

Varia

\section{Un culte domestique des Corybantes}

\section{Emmanuel Voutiras}

Édition électronique
URL : http://journals.openedition.org/kernos/1173

DOI : 10.4000/kernos. 1173

ISSN : 2034-7871

\section{Éditeur}

Centre international d'étude de la religion grecque antique

\section{Édition imprimée}

Date de publication : 1 janvier 1996

ISSN : 0776-3824

\section{Référence électronique}

Emmanuel Voutiras, "Un culte domestique des Corybantes », Kernos [En ligne], 9| 1996, mis en ligne le 21 avril 2011, consulté le 01 mai 2019. URL : http://journals.openedition.org/kernos/1173 ; DOI : 10.4000/kernos. 1173 


\section{Un culte domestique des Corybantes*}

En 1985, lors d'une fouille d'urgence sur la pente septentrionale de la table plate qui entoure la colline, habitée déjà à l'époque du bronze, connue sous le simple nom de Toumba (anciennement Toumba de Kalamaria) dans les environs de Thessalonique, $M$. Kostas Soueref a dégagé, dans un terrain surplombant le torrent qui délimite la colline vers le nord et l'ouest, des habitations des époques archaiqque et classique'. Les phases successives d'occupation s'échelonnent du Xe à la fin du IVe siècle av. J.-C. Ces maisons appartenaient à village qui fut abandonné dans le dernier quart du IVe siècle, sans doute lors de la fondation de la ville de Thessalonique par Cassandre, probablement au début de son règne, quand furent réunies dans un ample synécisme de nombreuses villes du pourtour du golfe thermaïque et de la Crousside ${ }^{2}$. La distance entre le bourg de Toumba et le tracé oriental des remparts de Thessalonique est à peine supérieur à $1 \mathrm{~km}$, tandis que la ville située sur le promontoire de Mikro Karabournou, qui est vraisemblablement l'ancienne Thermè $^{3}$, se trouve à $2,5 \mathrm{~km}$ à peine.

Une des ces maisons présente un intérêt particulier pour l'histoire de la religion grecque. Il s'agit d'une habitation spacieuse qui comprenait, dans sa dernière phase, attribuable au IVe siècle av. J.-C. ${ }^{4}$, plusieurs pièces (fig. 1). Une d'entre elles, assez grande (elle mesure $3,5 \mathrm{~m} \mathrm{x} 5 \mathrm{~m}$ ), contenait une baignoire qui se trouvait à gauche de l'entrée (fig. 2); elle communiquait avec une seconde pièce, un peu plus grande $(4 \mathrm{~m} \times 5 \mathrm{~m})$, où une fosse bordée de

Je tiens à remercier vivement $M$. Kostas Soueref pour son aide précieuse, ainsi que pour les clichés de sa foulle qui sont reproduits à la fin de cette étude. p. $235-46$.

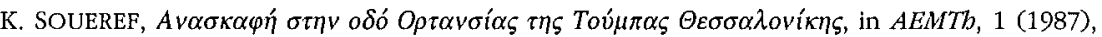

2 Sur la fondation de Thessalonique, qui doit dater de 316 ou 315 av. J.-C., voir en dernier lieu F. PAPAzOGloU, Les villes de Macédoine à l'époque romaine, Paris, 1988 (BCH Suppl. 16), p. 189190.

3 Le problème de l'identification de Thermè n'a pas encore été résolu de manière définitive; voir la discussion des témoignages antiques et des opinions divergeantes chez PAPAZOGLOU, op. cit.

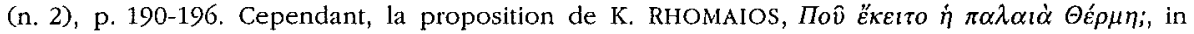
Makedonika, 1 (1940), p. 1-7, de situer cette ville sur le promontoire de Mikro Karabournou, à laquelle se sont ralliés N.G.L. HAMMOND, A History of Macedonia I, Oxford 1972, p. 150-152 et

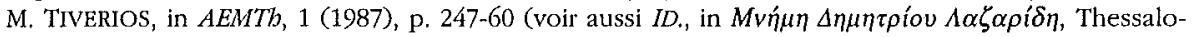
nique, 1990 , p. 71-81) reste la plus vraisemblable.

4 Pour une descripition détaillée de la maison et des phases d'occupation successives, voir SOUEREE, art. cit. (n. 1), p. 236-238. 
pierres plates servait probablement à écouler les eaux usées (fig. 3). Ailleurs dans la maison on a trouvé des objets d'usage quotidien : des meules, des mortiers et autres instruments pour traiter les aliments ainsi que des ustensiles de cuisine; des poids en terre cuite à attacher au bout des fils d'un métier à tisser. La maison possédait également un espace aménagé en cave, avec six pithoi enfoncés dans la terre où l'on entreposait le vin et probablement aussi l'huile (fig. 4). Le vin était sans doute un des principaux produits consommés dans cette maison, comme le montre une amphore trouvée dans le couloir, en face de l'entrée de la salle à la baignoire (fig. 5). La maison fut sans doute abandonnée, comme le reste du village, lors du synécisme de Thessalonique ${ }^{5}$. Il n'y a donc pas eu de destruction subite, et ceci explique le fait que les objets trouvés ne sont pas nombreux, les habitants ayant eu le temps d'emporter le mobilier. La date de l'abandon, probable en soi pour des raisons historiques, est confirmée par le fait que la dernière couche d'occupation contenait de la céramique du troisième quart du IVe siècle ainsi que des monnaies de Philippe II et d'Alexandre le Grand'.

Les trouvailles les plus intéressantes proviennent de la salle à la baignoire. Il faut bien remarquer qu'il ne peut s'agir d'une salle de bains ordinaire. $\grave{A}$ l'époque classique la baignoire était un objet qu'on ne rencontrait pas souvent dans les habitations privées ${ }^{7}$. Dans son étude minutieuse et approfondie des installations de bains dans la Grèce antique, René Ginouvès montre bien que la salle de bains telle que nous l'entendons semble ne prendre, au IVe siècle encore, une individualité que dans des cas assez rares et avec des différences de site à site : on ne connaît, à cette époque, que quelques exemples de maisons (notamment à Athènes et à Delphes) où une baignoire a été conservée en place, dans une pièce conçue manifestement pour la recevoir, et il faut dire qu'il s'agit toujours d'une salle de dimensions très réduites ${ }^{8}$. Dans le cas de Delphes la salle de bains est en plus liée au foyer de ce qui devait être la cuisine : c'est sans doute parce que le bain à la maison était normalement un bain chaud ${ }^{9}$. Contrairement à ces exemples, à Toumba, la salle dans laquelle se trouvait la baignoire ne ressemble pas à une salle de bains ordinaire : non seulement ses dimensions sont considérables, mais la céramique qu'elle contenait était, outre une petite cruche et une lampe, de qualité nettement supérieure à celle des autres pièces de la maison. Il y avait notamment des

\footnotetext{
5 Ceci est suggéré par l'absence de trouvailles postérieures au troisième quart du IVe siècle av. J.-C.

SOUEREF, art. cit. (n. 1), p. 237-238.

7 R. GINOUVÈs, Balaneutikè, Paris, 1962 (BEFAR, 200), p. 175. Un des plus anciens traités hippocratiques (Du régime dans les maladies alguës, éd. Litté II, p. 364) note combien il est rare qu'un malade puisse se baigner correctement chez lui.

8 GINOUVÈs, loc. cit. (n. 7). Cf. W. HÖPFNER et E.-L. SCHWANDNER, Haus und Stadt im Klassiscben Griecbenland. Neubearbeitung, München, 1994, p. 218, 320.

9 GINOUVÈs, loc. cit. (n. 7).
} 
coupes à boire. Parmi ces vases un petit canthare attique à vernis noir (fig. 6) du troisième quart du IVe siècle av. J.-C. ${ }^{10}$ porte une inscription gravée, que la forme des lettres situe également (malgré la gravure un peu hâtive) dans la

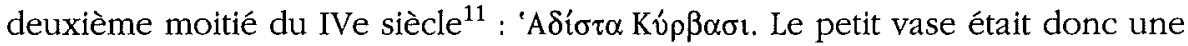
dédicace offerte par une femme nommée Adíc $\alpha^{12}$ aux Corybantes ${ }^{13}$, qui portent ici leur nom ancien, Kú $\beta \beta \alpha \tau \varepsilon \varsigma^{14}$. Cette offrande ${ }^{15}$ nous fournit donc la preuve que la salle contenant la baignoire était vouée à un usage particulier : il s'agissait d'un sanctuaire domestique où l'on pratiquait le culte des Corybantes. Des canthares votifs, dont un à inscription votive gravée, ont été trouvés dans le sanctuaire des Cabires de Thèbes ${ }^{16}$, dont le culte était vraisemblablement apparenté à celui des Corybantes ${ }^{17}$.

Les témoignages des auteurs anciens, bien qu'assez nombreux, contiennent des allusions plutôt que des renseignements précis au sujet de ces divinités et de leur culte ${ }^{18}$. Dans la mythologie, les Corybantes apparaissent comme des guerriers armés qui, avec leurs danses bruyantes au son du tambourin (instrument dont on leur attribuait l'invention) devant la grotte où fut élevé

10 Pour la provenance et la date $c f$. B. SPARKES - L. TALCOTT, The Atbenian Agora XII. Black and Plain Pottery, Princeton 1970, p. $122 \mathrm{n}^{\circ} 706-709$, pl. 28.

11 On notera en particulier le sigma angulaire formé en deux mouvements qui, comme l'a montré A. WILHELM, in $J O ̈ A I, 7$ (1904), p. 110-111, est fréquent à cette époque dans les tablettes d'imprécation et les lettres privées gravées sur des lamelles de plomb.

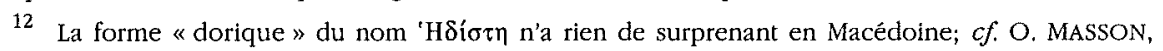
Quelques noms de femmes en Macédoine, in ZPE, 55 (1984), p. 133 (= Onomastica Graeca Selecta, II, p. 417). Le nom est attesté à Béroia et en Haute Macédoine; voir A. TATAKI, Ancient Beroea. Prosopography and Society, Athènes, 1988 (MEAETHMATA, 8), p. 354-355.

13 Les témoignages sur ces divinités ont été convenablement rassemblés par B. HEMBERG, Die Kabiren, Uppsala, 1950, p. 338-339; voir aussi la très bonne la discussion de F. GRAF, Nordiontscbe Kulte, Rome, 1985 (Bibllotbeca Helvetica Romana, 21), p. 319-334. Sur ce sujet voir aussi l'étude récente de Y. USTINOVA, Corybantism: The Nature and Role of an Ecstatic Cult in the Greek Polis, à paraître dans Horos, 10 (1996).

14 Voir HESYCHIUS s.v. ainsi que les remarques de GRAF, op. cit. (n. 13), p. 329-330. Le datif Kó $\rho \beta \alpha \sigma$ montre bien qu'il s'agit d'une inscription votive; $c f . B u l l . e ̂ p .1990, n^{\circ} 474$ (M. HATZOPOULOS). Le petit canthare a été publié par I. VOKOTOPOULOU dans le catalogue de l'exposition $L a$ civilisation grecque. Macédoine, royaume d'Alexandre le Grand, Marché Bonsecours, Montréal

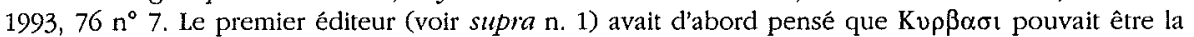
forme abrégée d'un patronyme Kvpß $\alpha$ ớov. Cette interprétation doit être abandonnée.

15 Les graffiti sur les vases grecs sont souvent des inscriptions votives; voir M. LANG, The Atbenian Agora XXI. Graffiti and Dipinti, Princeton, 1976, p. 52-53.

16 U. HeImberg, Das Kabirenbeiligtum bei Theben III. Die Keramlk des Kabirions, Berlin, 1982, p. 4-21. Pour le canthare inscrit voir G. BRUNS, in AA, 1964, p. 261 fig. 27; HEIMBERG, op. cit., p. $127 \mathrm{n}^{\circ} 9$, pl. 1.

17 C'est ce que suggèrent en premier lieu les trouvailles archéologiques; voir GRAF, $o p, c i t$. (n. 13), p. 328-329.

18 Il y a lieu de noter que le grand savant qu'était August LOBECK remarquait au sujet des Corybantes (Aglaophamos, Regimontii [Königsberg], 1829, p. 1139-1140) : Quamquam Corybantum nomen multorum memoratione celebratur; tamen, si res censenda est, praeter nomen nibil fere constat. C'est grâce aux trouvailles épigraphiques et archéologiques que notre connaissance de ce culte s'est élargie. 
l'enfant Zeus, couvrirent ses pleurs et le protégèrent ainsi de la voracité de son père Cronos $^{19}$. Ce mythe met en évidence un fait religieux dont nous aurons à parler, à savoir leur relation avec la Mère des dieux (divinité d'Asie Mineure assimilée volontiers à Rhéa, la mère de Zeus) ${ }^{20}$. Les Corybantes étaient d'ailleurs identifiés aux Courètes dès l'époque classique ${ }^{21}$; on les confondait en

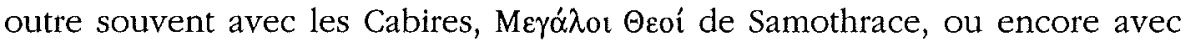
les Telchines, les Dactyles et même les Dioscures ${ }^{22}$.

Le témoignage du canthare inscrit jette une lumière inattendue sur les trouvailles de la salle de bains de Toumba et invite à les examiner en fonction de ce que nous savons sur le culte des Corybantes à l'époque classique. Les textes qui s'y réfèrent (en premier lieu certains passages très suggestifs de Platon) ont été rassemblés et interprétés par Ivan Linforth dans une étude claire et exhaustive ${ }^{23}$ : les cérémonies elles-mêmes ne sont pas décrites, mais leurs effets sur les participants se dégagent plus ou moins nettement. Les rites pratiqués sont souvent qualifiés de guérisons (ió $\mu \alpha \tau \alpha)$ et de purifications ( $\kappa(\theta \alpha \rho \mu o i)$. Nous apprenons que ceux qui y participaient entraient dans une sorte de transe, état que l'on désignait comme корv $\beta \alpha v \tau \imath \hat{\alpha} v$. La musique, surtout celle des auloi et des tambourins ainsi que la danse y jouaient un rôle

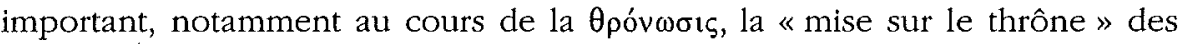
adeptes $^{24}$. Par ailleurs, l'emploi du verbe $\tau \varepsilon \lambda \varepsilon \hat{\imath} v$ et du mot $\tau \varepsilon \lambda \varepsilon \tau$ á à propos des rites des Corybantes montre que ceux-ci étaient des cérémonies d'un type

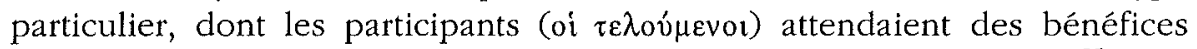
concrets, à savoir le soulagement de tensions émotionnelles ou mentales ${ }^{25}$.

Mais le document qui nous fournit les informations les plus précises en la matière est une inscription découverte vers le début de ce siècle à Érythrées et publiée par Wilamowitz en $1909^{26}$. Il s'agit d'un règlement relatif à un culte

19 Voir CALliM., Hymne à Zeus, 50-54; les témoignages sur le mythe de la naissance de Zeus ont été rassemblés par A.B. COOK, Zeus, I, Cambridge, 1915, p. 148-154.

20 F. CHAPOUTHIER, Les Dioscures au service d'une déesse. Étude d'iconographie religieuse, Paris, 1935 (BEFAR, 137), p. 168-172; HEMBERG, op. cit. (n. 13), 82-92; voir aussi infra, p. 249-250.

21 Voir EUR, Bacch., 120-134, avec le commentaire de E.R. DODDS, Euripides Baccbae, Oxford, 1960²; HEMBERG, op. cit. (n. 13), p. 296 n. 2; GRAF, op. cit. (n. 13), p. 324 n. 44.

22 Sur ses Identifications, les problèmes qu'elles posent et leur explication possible, voir HEMBERG, op. cit. (n. 13), p. 73-81; cf. GRAF, op. cit. (n. 13), p. 328-332. Le texte le plus explicite et le plus instructif en la matière est une longue digression dans le Xe livre de la Géograpbie de Strabon.

23 I. LINFORTH, The Corybantic Rites in Plato, in University of California Publications in Classical Pbilology, 13 (1946), p. 121-161.

24 Voir en particulier PLATON, Eutbyd., 277d-e; LINFORTH, art. cit. (n. 23), p. 122-124. Sur la $\theta$ póvwois, voir aussi K. KERÉNYI, Dionysos, Princeton, 1976, p. 263-265.

25 C'est ce que souligne à juste titre LINFORTH, art. cit. (n. 23), p. 155-57; voir aussi l'étude de Y. USTINOVA, supra n. 13.

26 U. v. WILAMOWITZ et P. JACOBSTHAL, Nordioniscbe Steine, in AbbAkBerlin (1909), p. 29-39. La dernière édition est celle de H. ENGELMANN et R. MERKELBACH, Inschriften von Erythrat und Klazomenai II, Bonn, 1972 (Inscbriften griechiscber Stadte aus Kleinasien, 2), $\mathrm{n}^{\circ} 206$. La pierre, conservée jadis au musée de l'École Évangélique de Smyrne, a malheureusement disparu dans 
public des Corybantes, auquel président deux prêtres nommés par la cité, un homme et une femme ${ }^{27}$ :

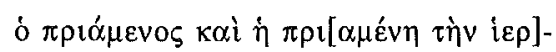

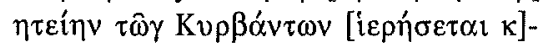

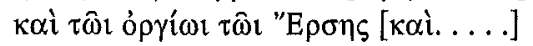

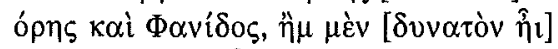

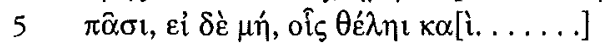

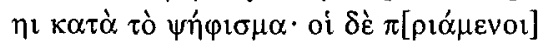

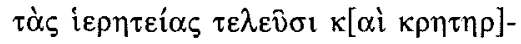

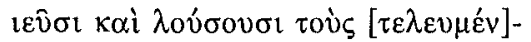

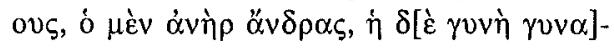

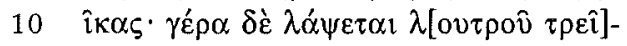

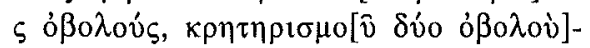

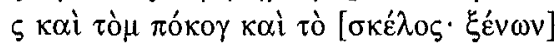

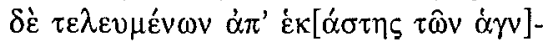

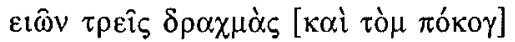

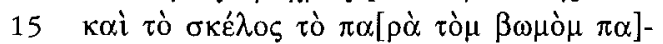

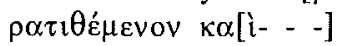

La prêtrise est obtenue, selon une pratique largement attestée dans les villes grecques d'Asie Mineure, par un acte d'achat public. Les rites que les deux prêtres avaient le devoir d'accomplir y sont énumérés : Il s'agissait du

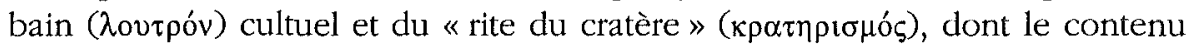
n'est pas clair, mais où le vin jouait sans doute un rôle important ${ }^{28}$. Ces rites étaient accompagnés de sacrifices, les prêtres obtenant une partie de la victime. Les participants étaient aussi consacrés au cours d'une cérémonie

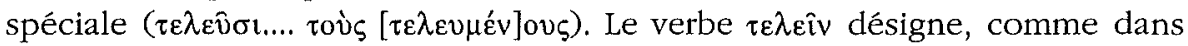
les textes évoqués ci-dessus (n. 24), l'ensemble des rites et les participants sont par conséquent appelés $\tau \varepsilon \lambda \varepsilon u ́ \mu \varepsilon v o r$. Le texte ne laisse aucun doute sur la nature de ces rites : c'étaient des purificatons ( $\left.\alpha \gamma v \varepsilon i \alpha \_\right)$. On les célébrait probablement de nuit, comme l'indique le témoignage de Démosthène $(18,249)$ au sujet d'une cérémonie du même type (voir ci-dessous).

La correspondance entre les actes cultuels mentionnés dans l'inscription d'Érythrées et les trouvailles de la maison de Toumba - plus particulièrement celles de la salle à la baignoire - est telle que celles-ci apparaissent comme une illustration du texte épigraphique. Les éléments essentiels sont, dans les deux cas, la pratique du bain et l'importance du vin. Les dimensions considérables de la salle et le fait qu'elle communiquait avec une autre, un peu plus grande,

l'incendie de 1922, mais un estampage est conservé dans les archives des $I G$ à Berlin (ENGELMANN et MERKELBACH, op. cit., pl. XXXVII) : le texte, attribuable à la deuxième moitié du IVe siècle, est gravé stoichédon en belles lettres régulières (28 par ligne) sur une stèle de calcaire local gris bleu.

27 Le texte est celui de ENGELMANN et MERKELBACH, loc. cit. Au sujet des rites évoqués voir en dernier lieu l'excellent commentaire de GRAF, op. cit. (n. 13), p. 320-325.

28 GRAF, op.cit. (n. 13), p. 320-321. 
indique que sa destination principale n'étaient pas les ablutions, mais les rassemblements, probablement cultuels, où plusieurs personnes participaient à des cérémonies religieuses, sans doute nocturnes, comme le suggère la présence d'une lampe. Le graffito votif sur le petit canthare fournit enfin la preuve que ce culte était bien celui des Corybantes.

La présence d'un sanctuaire dans une habitation privée n'a en soi rien de surprenant : les sanctuaires privés sont bien attestés en Grèce depuis l'époque achaïque. On y adorait pour la plupart les divinités tutélaires de la maison, Zeus Ktésios ou Herkeios, Hestia et Agathos Daimon (parfois sous la forme d'un serpent ${ }^{29}$. Mais il y avait aussi des hommes et des femmes, portant souvent le titre de prêtres, prêtresses ou devins, qui adoraient chez eux non pas des divinités tutélaires mais des dieux qui ne faisaient pas partie du panthéon officiel de la cité et dont le culte comportait souvent des rites d'initiation et de purification $^{30}$. Ces dieux étaient pour la plupart étrangers, ou présumés tels. C'est notamment le cas de la mère d'Eschine Glaucothéa, qui appartenait à une ancienne famille de devins ${ }^{31}$ et pratiquait chez elle de tels rites, comme nous l'apprend Démosthène dans le discours sur la couronne, en se gardant cependant de nommer la divinité en l'honneur de laquelle ils étaient célébrés; selon les scholiastes antiques il s'agirait du dieu phrygien Sabazios ${ }^{32}$. Quoi qu'il en soit, ces rites ressemblaient sans doute beaucoup à ceux des Corybantes, tels qu'on les pratiquait à Érythrées (Démosth., 18, 259) :

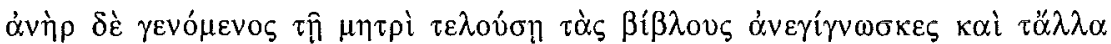

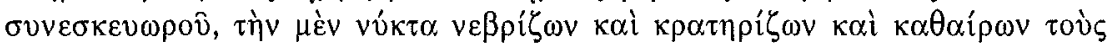

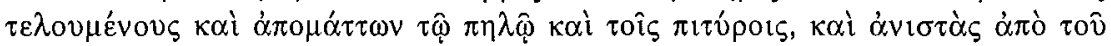

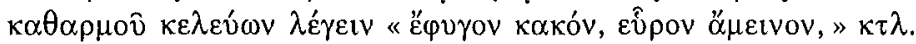

Devenu homme, pendant que ta mère pratiquait ses initiations, tu lui lisais les livres et tu participais à toutes ses machinations : pendant la nuit tu présentais aux initiés la peau de faon, tu leur donnais à boire le vin du cratère, tu les purifiais, tu les barbouillais de boue et de son; la purification terminée, tu les faisais lever et tu leur faisais dire : «J'ai échappé au mal, j’ai trouvé le bien » ${ }^{33}$.

29 M.P. NILSSON, Gescbichte der griecbiscben Religlon, $\mathrm{I}^{3}, 1967$, p. 402-406, 415; ID., Roman and Greek Domestic Cult, in Opuscula Romana, I (1954), p. 77-85 (= Opuscula Selecta III, Lund, 1960, p. 271-285).

30 Le caractère privé et mystique de ces cultes, dont celui des Corybantes, avait déjà été indiqué, en termes forts, par LOBECK, op. cit. (n. 18), I, p. 116; cf. LINFORTH, art. cit. (n. 23), p. 157.

31 Glaucothéa était sceur du devin Kléoboulos. Sur ce personnage, voir Chr. KAROUzos, $\Phi \rho o v \tau i \sigma \mu \alpha \tau \alpha$, in $\Theta E \Omega P I A . ~ F e s t s c b r i f t$ für W.-H. Schuchbardt, Baden-Baden, 1960, p. 113-122; cf. GRAF, op. cit. (n. 13), p. 321 n. 18.

32 GRAF, op. cit. (n. 13), p. 322 n. 24.

33 Trad. G. Mathieu (C.U.F.). La relation étroite entre ce texte et l'inscription d'Érythrées a été relevée par les commentateurs; voir H. WANKEL, IErytbrat 206,6-12 und Demostb. 18,259, in ZPE, 34 (1979), p. 79-80. 
Démosthène, sachant bien que nombreux étaient ceux qui voyaient d'un mauvais œil les cultes étrangers pratiqués en privé, n'a pas manqué l'occasion de railler son adversaire en lui imputant d'avoir été l'aide de sa mère dans des cérémonies de ce genre. La méfiance publique à l'égard des cultes mystiques d'origine orientale, dont les fidèles dansaient au son de l'aulos et du tambourin, est bien décrite dans les Bacchantes d'Euripide, où Dionysos est présenté comme le fondateur d'un pareil mouvement religieux ${ }^{34}$. La description du rituel montre d'ailleurs que des éléments dionysiaques étaient bien présents dans le culte pratiqué par Glaucothéa ${ }^{35}$. Même si Démosthène a réuni, par ironie, des traits disparates (ce qui ne semble pas être le cas), la ressemblance avec les rites des Corybantes mentionnés dans l'inscription d'Érythrées montre bien qu'il énumère des cérémonies bien connues du public athénien. Les rites mystiques et purificatoires jouissaient en effet d'une certaine popularité, car ils permettaient de calmer les angoisses et les troubles mentaux. Nombreux étaient sans doute ceux qui y cherchaient une cure pour les obsessions et les désordres psychologiques, tel Bdelykléon dans les Guêpes d'Aristophane, 119-120, qui, dans son vain effort de guérir son père Philokléon de l'obsession des procès, l'avait soumis, entre autres, aux rites purificatoires des Corybantes.

Les Grecs de l'époque classique attribuaient volontiers au culte des Corybantes ainsi qu'à d'autres du même genre une origine orientale. Cette provenance n'est en fait souvent qu'un moyen d'indiquer la nature orgiaque de ces cultes ${ }^{36}$. Elle s'exprime surtout par le rattachement de certaines divinités associées à la musique et aux danses extatiques comme les Courètes et les Pans (au pluriel), gardiennes des portes et des passages comme Hécate et Hermès, ou présidant à des mystères comme Perséphone et Dionysos - au cercle de la Mère des dieux (ou Cybèle) ${ }^{37}$, déesse orientale vénérée sous plusieurs noms, dont le culte, originaire d'Asie Mineure, envahit la Grèce à la fin du Ve et au IVe siècle av. J.-C. Les Corybantes n'ont probablement pas échappé à cette règle ${ }^{38}$, même si leur culte n'est nulle part directement associé à celui de la Mère des dieux ${ }^{39}$. En faveur de cette association on peut évoquer, entre autres monuments, un relief votif fragmentaire trouvé non loin de Thessalonique, à Poteidaia, conservé au musée de Polygyros (fig. 7), à peu près

34 DODDS, op. cit. (n. 21), introduction p. XX-XXII.

35 GRAF, op.cit. (n. 13), p. 322-323.

36 I.M. LEWIS, Ecstatic Religion, Harmodsworth, 1971, p. 101; cf. GRAF, op. cit. (n. 13), p. 288 et n. 43.

37 O. WALTER, KOYPHTIKH TPIA , in JÖAI, 31 (1938-39), p. 53-80 : excellente étude d'ensemble sur les représentations des Courètes sur les reliefs votifs.

38 E.R. DODDS, The Greeks and the Irrational, Berkeley - Los Angeles, 1951, p. 96 n. 90; LINFORTH, ait. cit. (n. 23), p. 157.

39 Cf. GRAF, op. cit. (n. 13), p. 331. 
contemporain du petit canthare de Toumba ${ }^{40}$. On y voit, devant une famille d'adorants, un jeune homme nu portant casque et bouclier dans une attitude de danse, que sa taille plus grande identifie comme un personnage divin. Le guerrier faisait sans doute partie d'une triade de guerriers dansants, Courètes ou Corybantes. La composition du relief, dont il manque un peu plus de la moitié, doit être sans doute complétée avec une Cybèle assise, comme le suggèrent les parallèles iconographiques ${ }^{41}$. Sur un autre relief votif en forme de naïskos, provenant d'Amphipolis et conservé à Thessalonique, les trois guerriers sont représentés dans le fronton ${ }^{42}$. Qu'il s'agisse des Courètes ou des Corybantes, le culte de ces guerriers danseurs était sans doute répandu sur toute la côte nord de la mer Égée ${ }^{43}$.

Les cultes mystiques qui comportaient des rites d'initiation et de purification pouvaient être perçus comme une menace à la cohésion de la cité, surtout quand leur pratique était le fait de groupes privés échappant à tout contrôle. Ils pouvaient donc faire l'objet de mesures restrictives, voire répressives. Il suffit de rappeler la fameuse affaire des bacchanales à Rome. Ce sont sans doute les cultes de ce genre que vise Platon, quand, légiférant pour sa cité des Magnètes, il interdit les sanctuaires et cultes privés; car il a autorisé ailleurs (Lois, 717b) les rites accomplis selon la loi pour les dieux domestiques dans des sanctuaires privés $^{44}$. Il propose donc la loi suivante (Lois, 910b-c):

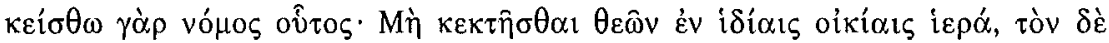

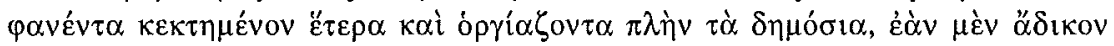

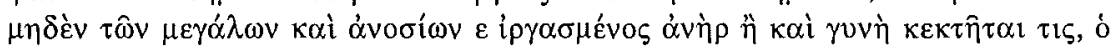

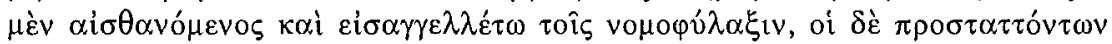

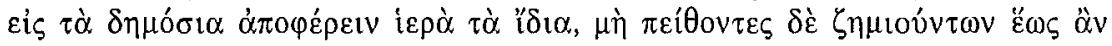
$\dot{\alpha} \pi \varepsilon v \varepsilon \chi \theta \hat{n}$.

Voici la loi que le législateur établira : pas de sanctuaires de dieux dans les maisons privées. Si quelqu'un est convaincu d'en avoir d'autres que les sanctuaires publics et d'y faire ses dévotions (ópyí $\zeta_{\text {ov }} \tau \alpha$ ), sans que, homme ou femme, il ait dans son passé aucune injustice ou impiété grave, quiconque en aura connaissance devra le dénoncer aux gardiens des lois; ceux-ci lui ordon-

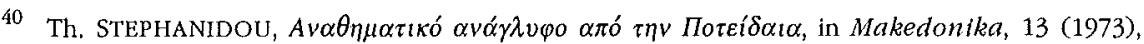
p. 106-115, pl. I. Le relief peut être daté par son style dans les années '30 du IVe siècle av. J.-C.; c'est une œuvre attique, comme l'indiquent le style et le matériel, qui est du marbre du Pentélique.

41 STEPHANIDOU, ait. cit. (n. 40), p. 111-115.

42 STEPHANIDOU, art. cit. (n. 40), p. 113, pl. III.

43 Cf. HEMBERG, op. cit. (n. 13), chap. II.

44 A.J. FESTUGIÈRE, L' Épinomis» et l'introduction de cultes étrangers à Atbènes, in Conjectanea Neotestamentica (1947), p. 72-74 (= Ëtudes de religion grecque et bellênistique, Paris 1972, p. 135-137). 
neront de transporter dans un temple public son sanctuaire privé, et s'il désobéit, le mettront à l'amende jusqu'à ce qu'il ait fait ce transfert ${ }^{45}$.

Cette loi n'est pas conçue pour une cité idéale; elle correspond tout à fait aux réalités de l'époque : la fondation d'un sanctuaire public destiné à recevoir les cultes privés d'un clan, celui des Clytides dans l'île de Chios, nous est connue par une inscription du troisième quart du IVe siècle ${ }^{46}$.

À Érythrées aussi le culte des Corybantes était, semble-t-il, célébré dans des cérémonies privées avant d'être soumis au contrôle de la cité. C'est ce que précise un document nouveau, une inscription du musée universitaire de Bonn, que publiera $N$. Himmelmann ${ }^{47}$. Ce texte est le décret de la ville qui règle la pratique du culte des Corybantes auquel le règlement que nous avons étudié fait allusion (1. $6: \kappa \alpha \tau \dot{\alpha} \tau o ̀ ~ \psi \eta ́ n \varphi \sigma \mu \alpha)$. La rémunération des prêtres officiels dans les sacrifices publics et privés y est précisée. Il contient aussi une disposition selon laquelle des prêtres et des prêtresses autres que ceux nommés par la cité ont le droit d'accomplir les mêmes rites (l'initiation, le bain cultuel et le rite du cratère) à condition de donner aux prêtres officiels la moitié de leurs recettes. La liberté de choisir indique que d'anciens prêtres privés ont continué leur pratique. Une situation analogue existe dans les thiases de Dionysos à $\operatorname{Cos}^{48}$ et à Milet $^{49}$. On peut en conclure que les cités cherchant à réglementer et à contrôler les cultes mystiques ou purificatoires pratiqués jusqu'alors par des particuliers, se sont vues obligées, dans un premier temps, de tenir compte d'anciennes traditions familiales. Dans le cas de la bourgade de Toumba, le culte des Corybantes était sans doute resté un culte domestique; on peut supposer qu'il fut transféré à Thessalonique lors du synécisme et placé peutêtre, comme à Érythrées, sous le contôle de la citée $e^{50}$.

La trouvaille de 1985 n'est pas unique. Dans le même bourg de Toumba K. Soueref a trouvé, en 1989, une autre maison avec une salle à baignoire, semblable par les dimensions et le dispositif à celle que nous avons considé-

45 Trad. A. Diès (C.U.F.).

46 GRAF, op. cit. (n. 13), p. 428-29, I.Ch. 3.

47 Voir provisoirement N. HIMMELMANN, Antike zwischen Kommerz und Wissenschaft. 25 Jabre Erwerbungen fil das Akademiscbe Kunstmuseum Bonn, in Nordrbein-Westfaliscbe Akademie der Wissenscbaften. Vortralge G 326 (1994), p. 24, pl. 1. Il s'agit d'un fragment de calcaire gris bleu, appartenant probablement à la même stèle qui portait l'inscription d'Érythrées que nous avons examinée (supra, n. 26): le texte est gravé stoichédon avec 28 lettres par ligne et la grandeur des lettres est à peu près la même.

$48 S y l l{ }^{3}, 1012$. F. SOKOLOWSKI, Lois sacrées des cités grecques, Paris, 1969, n 166.

49 F. SOKOLOWSKI, Lots sacrées d'Asie Mineure, Paris, 1955, $\mathrm{n}^{\circ} 48,18$.

50 Un culte des Cabires (ou Corybantes) est en effet attesté à Thessalonique par CLÉMENT D'AlEXANDRIE, Protrept., II, 19, 1-3; les témoignages antiques ont été rassemblés et discutés par HEMBERG, op. cit. (n. 13), p. 205-210. Il est tentant de supposer que ce culte se rattache à celui du IVe siècle av. J.-C. Dans ce cas, la trouvaille de Toumba infirmerait l'opinion de GRAF, op. cit. (n. 13), p. 331, selon qui ce culte ne paraît pas être ancien (kaum alteingesessen). 
rée ${ }^{51}$. S'agirait-il d'un deuxième sanctuaire privé où l'on pratiquait les mêmes rites ? Malheureusement les trouvailles, banales et peu nombreuses, ne permettent pas de trancher. Mais l'analogie avec le sanctuaire domestique des Corybantes identifié par le canthare inscrit est évidente. Dans les deux cas l'eau jouait un rôle important. On a donc le droit de se demander si les purifications rituelles n'avaient pas une place importante dans la petite bourgade de Toumba, et, partant, de la cité dont elle dépendait. Ceci peut être mis en relation avec le nom de cette cité, Thermé, qui dénote sans doute la présence d'eaux chaudes curatives ${ }^{52}$. Quoi qu'il en soit, le culte des Corybantes dont nous avons pu étudier les traces matérielles s'insère fort bien dans ce que les écrivains et les inscriptions nous apprennent à ce sujet. Des témoignages convergents indiquent qu'il s'agissait d'un culte assez répandu et populaire, pratiqué surtout en privé. Les règlements d'Érythrées montrent de quelle façon les cités ont cherché à le contrôler et à l'insérer dans le cadre des cultes civiques.

Université de Thessalonique

Emmanuel VOUTIRAS

Département d'archéologie et d'histoire de l'art

GR - 54006 THESSALONIQUE

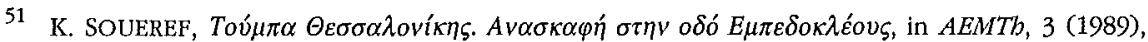
p. $215-220$.

52 Sur les diverses opinions émises à ce sujet voir en dernier lieu PAPAZOGLOU, op. cit. (n. 2), p. 191-192. Une inscription inédite, copiée par L. Robert au musée de Thessalonique en 1932 (L. ROBERT, in $R P b$ [1974], p. 220-221; cf. PAPAZOGLOU, loc. cit., n. 33) mentionne des eaux

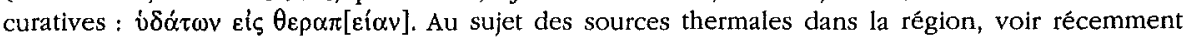
P. NIGDÉLIS, Auli Avit: elne Italikerfamilie in Thassalonike, in TEKMHPIA, 1 (1995), p. 47-65 (en grec moderne avec résumé en allemand), qui publie une nouvelle inscription où apparaît le mot $\theta \varepsilon \rho \mu \alpha ́$. 


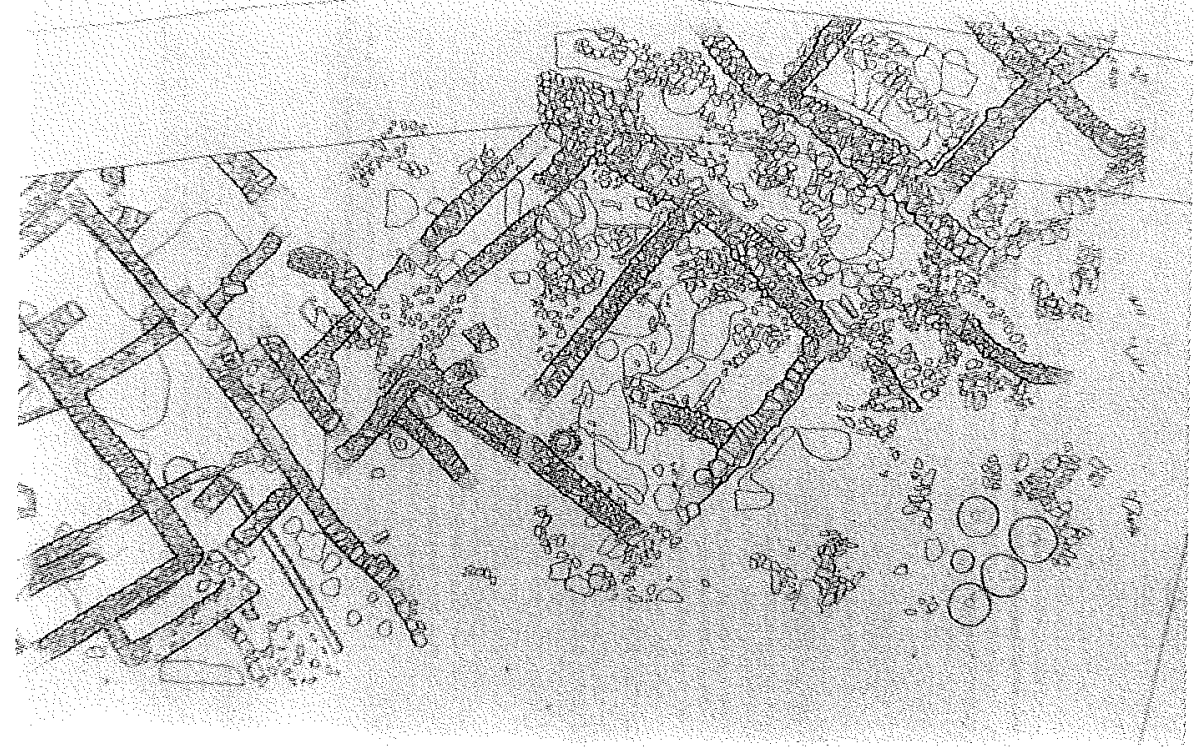

Fig. 1 : Thessalonique, Toumba : maison du IVe siècle av. J.-C.

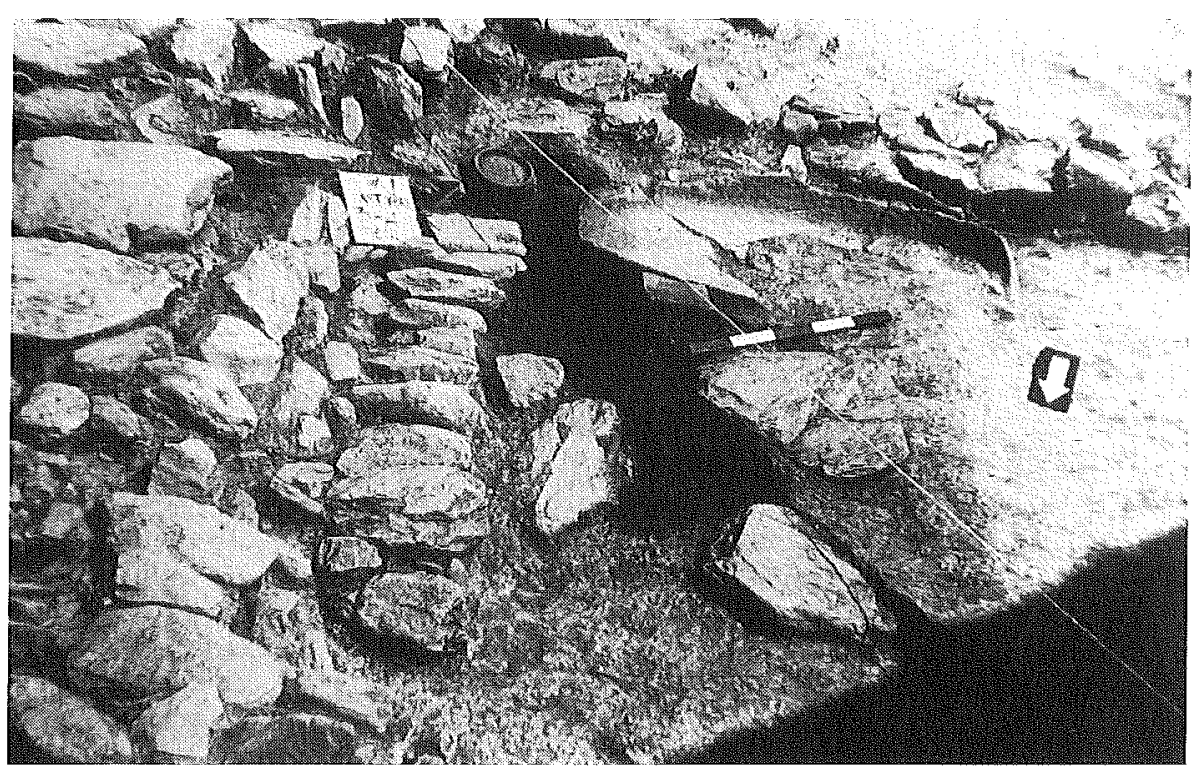

Fig. 2 : Thessalonique, Toumba : restes de baignoire dans la maison fig. 1. 


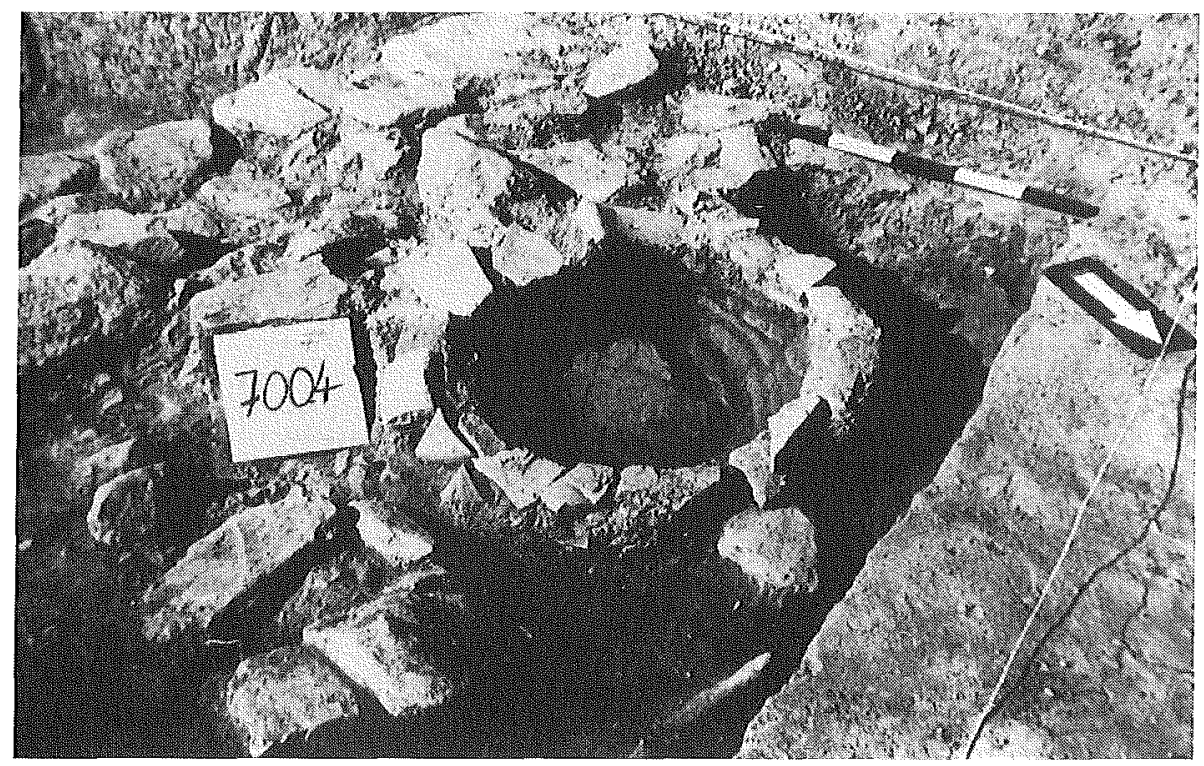

Fig. 3 : Thessalonique, Toumba : fosse pour l'écoulement des eaux usées dans la maison fig. 1.



Fig. 4 : Thessalonique, Toumba : pithoi enfoncés dans le sol de la maison fig. 1. 


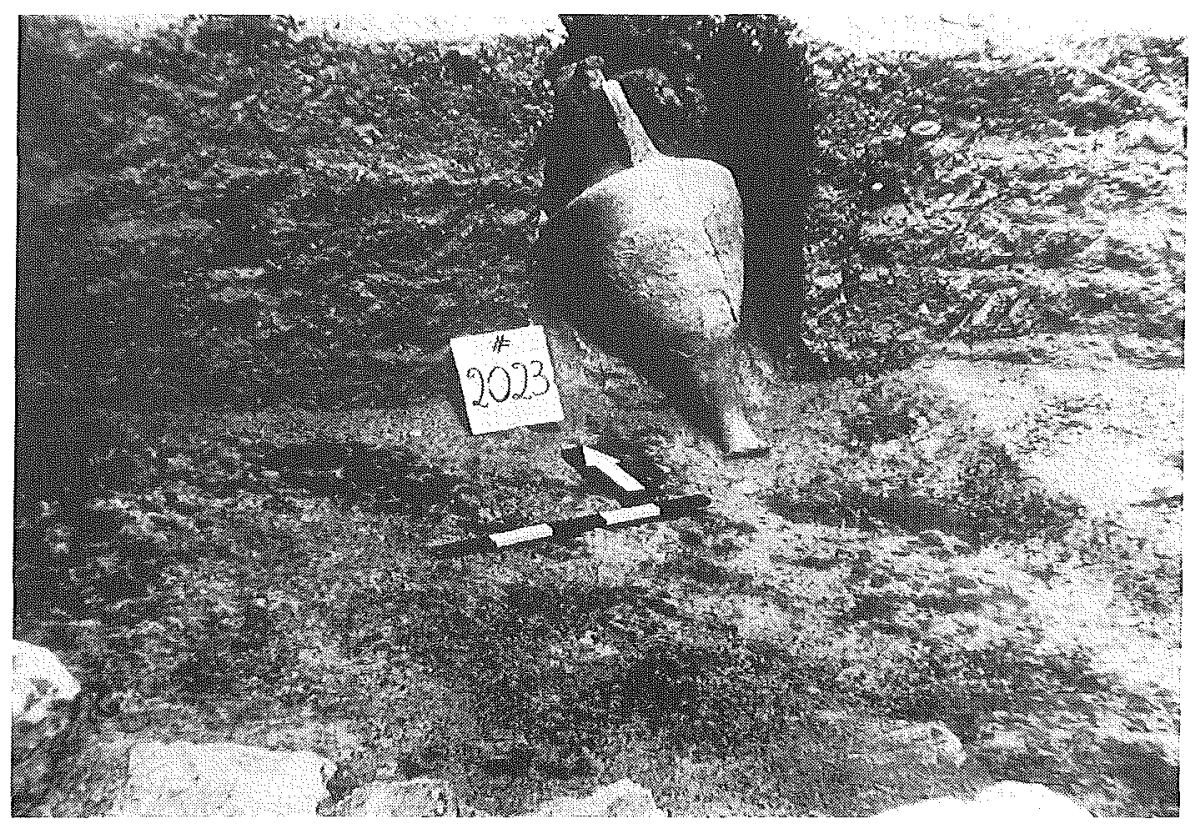

Fig. 5 : Thessalonique, Toumba : amphore à vin trouvée dans le corridor de la maison fig. 1.

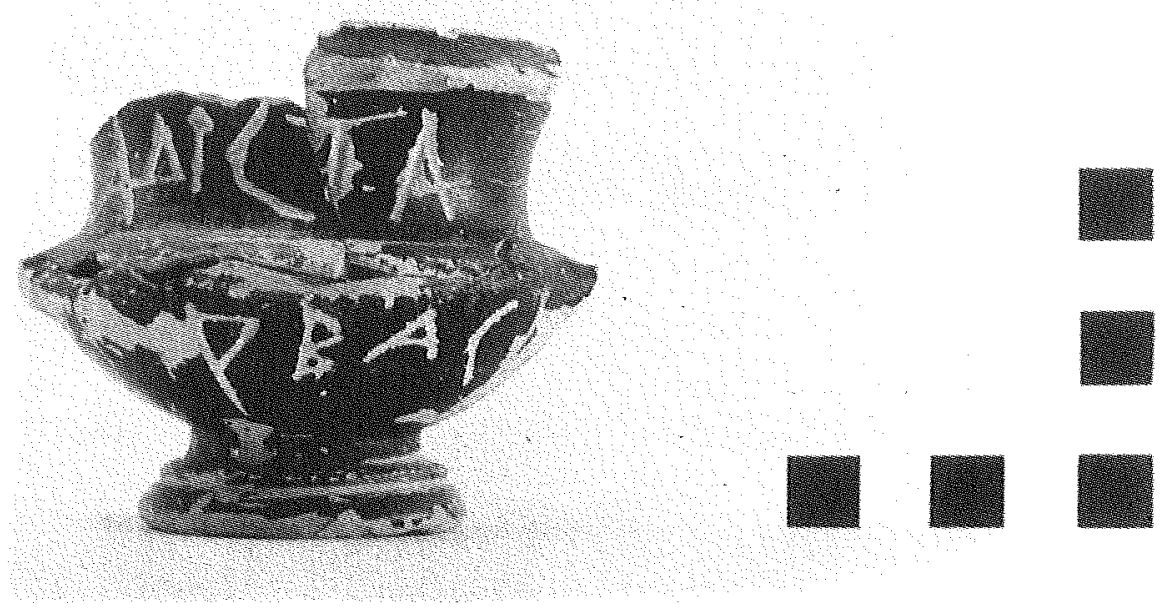

Fig. 6 : Thessalonique, Toumba : petit canthare inscrit (Thessalonique, Musée Archéologique). 


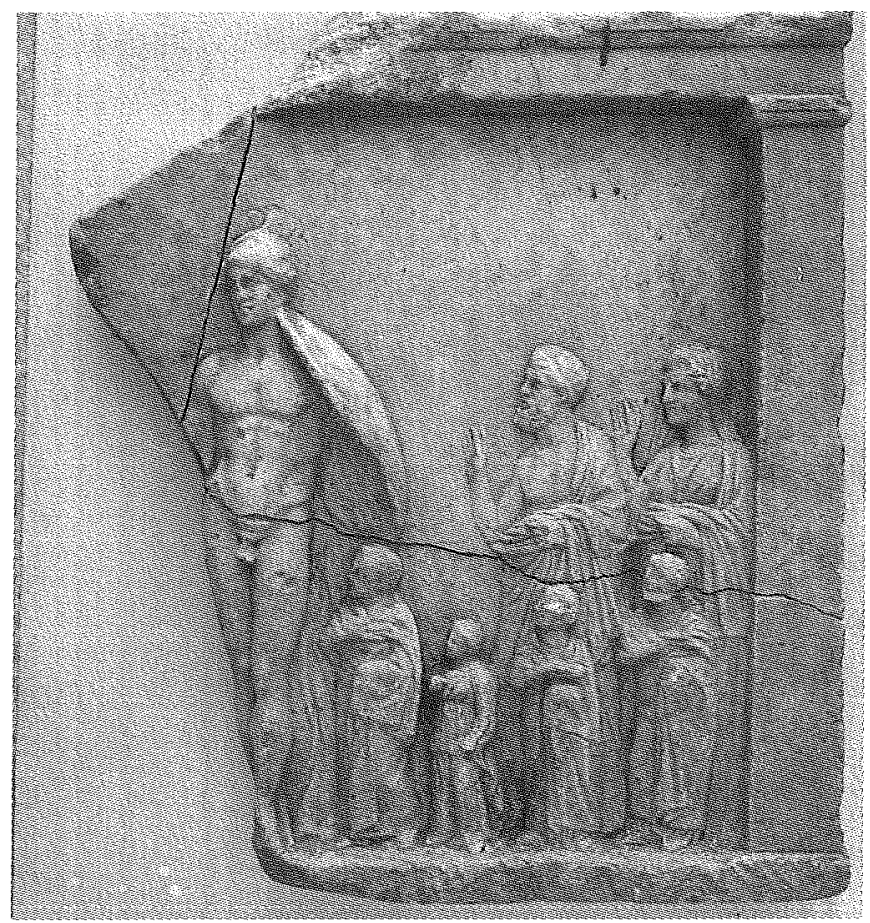

Fig. 7 : Relief votif aux Corybantes ou Courètes trouvé à Potidée (Polygyros, Musée). 